\title{
Construction of the Framework for Exploring the Method of Air Pollution Control in Urban Planning
}

\author{
Jiang Tao, Wei Lina, Bai Li \\ East University of Heilongjiang, Harbin City, Heilongjiang Province, 150080
}

\begin{abstract}
In recent years, with the rapid development of the economy and the continuous advancement of the urbanization process and the continuous deepening of the degree of industrialization, the problem of air pollution is rapidly expanding in cities. Especially since 2013, a large-scale smog problem has appeared in the northern part of my country. The problem of air pollution has received widespread attention from the state and society, and has now become an important influencing factor restricting the construction of ecological civilization in my country. Therefore, through the analysis of atmospheric pollutants and the guidance of urban planning, we can fully optimize and distribute the industrial structure and deal with atmospheric problems from the source.
\end{abstract}

\section{Introduction}

Urban planning plays an extremely important role in guiding the construction and development of cities. However, due to the long-term lack of awareness of environmental protection and the lack of guidance by the state on the current economic and social development, we have neglected the protection of the environment during the economic development of cities. But in recent years, due to severe smog in many places in my country, it has had a serious adverse impact on people's health and daily life. Haze weather has now become a typical environmental disaster event, which is seriously affecting the achievement of my country's ecological and social goals. Therefore, we must take environmental issues into consideration when carrying out urban development goals and urban planning, and conduct special investigations to plan against them [1].

\section{2 the Impact, Harm and Treatment of Urban Air Pollution}

\subsection{Effects and Hazards of Atmospheric Pollutants}

Typical air pollutants are diverse, generally including particulate matter, liquid aerosol, nitrogen oxides, sulfur oxides, volatile organic pollutants, etc. [2]. Therefore, we take volatile organic pollutants as an example to briefly introduce the sources, effects and hazards of similar pollutants. In recent years, with the rapid development of my country's urbanization and industrialization, volatile organic compounds (Volatile organic compounds, VOCs) have attracted more and more scientific researchers' attention due to their large hazards and wide sources. In 1989, the World Health Organization (World Health Organization, WHO) defined VOCs as a general term for organic compounds other than insecticides with boiling points between $50-260^{\circ} \mathrm{C}$ and a saturated vapor pressure greater than $133.322 \mathrm{~Pa}$ at room temperature. Its main components are hydrocarbons, oxyhydrocarbons, halogen-containing hydrocarbons, nitrogen hydrocarbons, sulfur hydrocarbons, and low-boiling polycyclic aromatic hydrocarbons, etc. It is a type of organic pollutant that is ubiquitous and complex in indoor and outdoor air.

Table 1 Source Details of VOCs

\begin{tabular}{|c|c|c|c|c|}
\hline Source & $\begin{array}{c}\text { Industrial } \\
\text { Production }\end{array}$ & Transportation & $\begin{array}{c}\text { Organic } \\
\text { Matter } \\
\text { Burning }\end{array}$ & Other Methods \\
\hline Production Ratio & $45.7 \%$ & $30.9 \%$ & $13.5 \%$ & $9.9 \%$ \\
\hline
\end{tabular}

As shown in Table 1, volatile organic compounds are mainly produced in industrial production, transportation, and organic matter combustion. Among them, industrial production $(45.7 \%)$ has the highest proportion, followed 
by transportation $(30.9 \%$ ), and organic substances (such as wood, tobacco, gasoline, etc.) will also produce volatile organic compounds when they are not completely burned. As can be seen from the table, in terms of pollution sources, industrial production and transportation account for a relatively large amount, and the sum of the two accounts for $76.6 \%$ of the total emissions. It is a major polluting industry, and this is also an important goal for pollution prevention in the next stage. Car exhaust pollution is an important source of outdoor pollution. In addition, wall varnishes, organic paints, decorative boards and other interior decoration and decoration materials can release formaldehyde, aromatic hydrocarbons, toluene, ethylbenzene, benzene, acetone, acetaldehyde and other volatile organic substances at room temperature. Daily chemicals such as detergents, bleaching powders, softeners and detergents can also cause hydrocarbons, aromatic hydrocarbons and chlorinated hydrocarbons to be released into the atmosphere. Among the man-made pollution sources, there is one type of solvent that people are most exposed to. In addition to making spray paint or thinning paint, it can also be used to dissolve resin, rubber and other adhesives. The other type is hydrocarbons and halogenated hydrocarbons, such as internal combustion engine fuels, dichloromethane, injection tanks, etc. filled with freon type injections. Due to the wide variety of these solvents and the complex composition, the toxicity and irritation they exhibit can be carcinogenic, teratogenic, mutagenic, etc. And it will also cause great harm to the environment, the growth of animals and plants and human health. Therefore, studying the existence, source, distribution, migration and transformation of these pollutants in the environment and their impact on human health has always been paid attention to and become the focus of research at home and abroad.

With the improvement of people's living standards in my country, people's requirements for quality of life are getting higher and higher, and the awareness of self-protection has also increased. People are beginning to realize that high-quality air is the guarantee of people's health. However, the huge changes in the structure of buildings have led to the extensive use of new building materials, thermal insulation materials, sealing materials, etc., and the widespread use of deodorants, insecticides, and various cleaning supplies in homes. These materials are releasing mixed organic compounds, which severely damage the indoor air quality, which often leads to symptoms such as fatigue, drowsiness, headache, respiratory diseases, itchy skin and allergies. VOCs are not only harmful to humans, but also harmful to the atmosphere. Its harm to the atmospheric environment is mainly because it promotes the formation of ozone-type photochemical oxidants. VOCs not only undergo photochemical reactions in sunlight, but also increase the concentration of the ozone layer and exacerbate the greenhouse effect. Moreover, under certain meteorological and geographical conditions, photochemical smog will also be formed, which will cause serious damage to the surrounding plants and increase the respiratory disease of the human body. In addition, the degree of pollution varies depending on the location in the atmosphere and the nature of the species.

\subsection{Governance}

There are usually two goals for the disposal of atmospheric pollutants, namely, while protecting the environment, trying to harmlessly recycle or recycle the pollutants. At present, for these complex air pollutants, the world has developed different means and methods to deal with them. At present, domestic and foreign control methods for atmospheric pollutants are mainly divided into two types, namely, adsorption recovery method and destruction method. According to the different types of pollutants, concentration and flow rate, different disposal methods are adopted. Generally speaking, the recycling method is suitable for pollutants with high economic value and high concentration, which can minimize production losses and have greater economic benefits. However, the destruction rule is mainly for low-concentration, mixed pollutants. The pollutants under this condition are difficult to be recovered, and they can only be detoxified in a harmless manner to minimize the harm to the environment.

Table 2 Comparison of Commonly Used Adsorption and Recovery Technologies

\begin{tabular}{|c|c|c|c|}
\hline $\begin{array}{c}\text { Adsorption Recovery } \\
\text { Technology }\end{array}$ & Adsorption Method & Condensation Method & Membrane Separation \\
\hline Purification Efficiency & $75 \%-85 \%$ & $75 \%-80 \%$ & $80 \%-90 \%$ \\
\hline Application Cost & Medium & Higher & High \\
\hline Application Field & Petrochemical & $\begin{array}{c}\text { Pharmaceutical, } \\
\text { Chemical }\end{array}$ & $\begin{array}{c}\text { Multi-component } \\
\text { Contaminants }\end{array}$ \\
\hline Security & Higher & Low & Lower \\
\hline
\end{tabular}

As shown in Table 2, adsorption recovery technologies include adsorption method, membrane separation method and condensation method. This type of treatment does not damage the pollutants. Its advantage lies in the recovery of high-concentration pollutants of economic value, but it is also accompanied by the risk of secondary pollution. It can be seen from the table: (1) In terms of purification efficiency, the membrane separation method is based on the mass transfer rate of each component of the pollutant. Under the pressure of the pressure, it is separated by selective permeation of the membrane. Therefore, its' purification efficiency is the highest; (2) In terms of application cost, the adsorption method is mature and can be recycled, the cost is higher than the other two methods, and it has higher economy; (3) From the perspective of safety, the condensation method requirements are higher, the 
content of the extracted pollutants is often within the explosion limit, and the risk is higher. Therefore, its safety is at the lowest level, and the adsorbent selected by the adsorption method (activated carbon, zeolite, metal organic framework, etc.) has strong stability and belongs to the most safe reagent type. In summary, from the analysis of various data indicators, it can be seen that in the process of pollutant treatment, the comprehensive effect is adsorption method $>$ membrane separation method $>$ condensation method, and we need to choose according to the actual situation.

The method of destroying low-level atmospheric pollutants is to convert atmospheric pollutants into chemically harmless compounds through chemical means. The technology can be subdivided into the following types: (1) Low-temperature plasma technology, which uses a high-voltage electric field to discharge the medium to form ions, groups, free radicals and other unstable reactive substances and pollutant molecules to contact each other to achieve the purpose of the pollutants activation and degradation. At present, low-temperature plasma technology is widely studied due to the advantages of simple operation process, it can be carried out at normal temperature and pressure, small space requirements for equipment and high efficiency of pollutant treatment; (2) Catalytic combustion technology, the use of catalysts can effectively change the reaction path. The technology of reducing the activation energy and accelerating the reaction rate, reducing the temperature required for the oxidation of pollutants, and catalyzing the oxidation to non-hazardous substances at low temperatures $(200 \mathrm{oC}-400 \mathrm{oC})$. The goal is to completely destroy the pollutants, rather than recovering them like the adsorption method. Compared with traditional direct combustion technology, catalytic combustion has lower cost, simple technical equipment, and high thermal efficiency; (3) Photocatalytic technology, which can use semiconductor catalysts to accelerate the reaction under light conditions. Because photocatalytic technology can directly use inexhaustible solar energy to degrade different kinds of pollutants, and can degrade pollutants through the use of light energy, this technology has received extensive attention in recent years. Unlike the other methods mentioned above, photocatalysis is friendly and non-toxic to the human body and the environment due to the small space required for the reaction and the mild reaction conditions. It is more suitable for the treatment of indoor pollutants, such as formaldehyde and other pollutants.

Based on the vigorous development of pollutant disposal methods in recent years, it is expected to achieve effective control of atmospheric pollutants through the selection and reasonable combination of pollution control technologies in the process of urban planning.

\section{Guiding Suggestions for Urban Planning on Air Pollution Control}

At present, according to the existing survey, due to the lack of reasonable guidance for urban planning, my country's urban air quality problems are particularly serious. As my country's air quality has entered a large-scale compound pollution stage, it has also brought serious adverse reactions. However, if the city planning can now be prepared rationally, under the guidance of scientific city planning, the industrial structure will be optimized and the sources of pollution will be properly placed. At the same time, the city is divided into functional areas, and the analysis of atmospheric pollution sources and the evaluation of urban pollution capacity allow us to avoid hazards as much as possible in future development. Fundamentally further prevent the further deterioration of the atmospheric environment, which is more conducive to the maintenance and protection of the atmospheric environment $[3,4]$.

\subsection{Adhere to the Basic Policy of Sustainable Development}

In the current urbanization process, most cities do not meet the requirements of reform and opening up. The industrial layout of most cities is unreasonable, and the city's infrastructure is lagging behind, which is far behind the current level of urbanization development, which directly leads to the current situation of frequent environmental problems. The reason is mainly because these cities were influenced by the early unreasonable and unscientific thinking of "urban construction must be produced before life". In the past ten years, although China has made remarkable achievements in the related fields of sustainable development such as energy conservation and emission reduction, population control and related fields. However, the current situation of high consumption, high pollution, low return and low output in urban development has not been fundamentally changed. It can be seen that sustainable development is the most basic development strategy in China, and the sustainable development strategy must be strictly adhered to for a long time as a guideline in urban planning.

\subsection{Existing Air Pollution Problem Planning}

In view of the existing pollution control problems, the author has described in detail in the previous chapter. In today's urban planning, we can no longer avoid the harm and impact of environmental problems caused in the past. Therefore, we need to analyze and control the existing air pollution problems. We need to adopt appropriate scientific and technological remediation methods by introducing advanced science and technology in the corresponding high environmental pollution damage areas. At the same time, when reducing pollutant emissions, we should vigorously advocate a new concept of green living and reduce the secondary damage to the environment caused by residual environmental pollutants.

\subsection{Optimize the Industrial Structure}

Judging from the current global form, ensuring economic 
development will inevitably put a certain pressure on the environment. However, different industries clearly have different environmental demands, so the impact of industrial structure control on the environment is self-evident. In addition, environmental issues have obvious regional characteristics, which further illustrates the importance of urban planning.

The main purpose of industrial optimization of cities is to control the source of atmospheric pollutants. Industrial structure directly affects the difficulty of environmental protection. Obviously, different industries have different pressures on environmental protection. Generally speaking, secondary industries, such as high-pollution industries such as chemicals and energy, have great pressures on environmental protection. In contrast, the potential pressure of ththe development of tertiary industriese for environment is small. In a reasonable urban planning, we can't place industries that have a greater impact on the atmospheric environment in the high-function areas of the city, but we must minimize their environmental impact and place them in low-function areas or the smaller area which has less impact to high-function areas. A certain region implemented a new industrial structure adjustment plan in early 2017, and then conducted data monitoring on the concentration of common pollutants throughout the year, and compiled and plotted the data to obtain Figure 1. It can be drawn from Figure 1 that the pollutants NO2 $\left(63.6 \mathrm{mg} / \mathrm{m}^{3}\right)$, SO2 $\left(52.3 \mathrm{mg} / \mathrm{m}^{3}\right)$, PM10 $\left(60.3 \mathrm{mg} / \mathrm{m}^{3}\right)$, PM2.5 $\left(73.5 \mathrm{mg} / \mathrm{m}^{3}\right)$ reached their peaks in January 2017 Although there were fluctuations throughout the year, the overall development has followed a downward trend. The main reason for the peak of ozone in June is that volatile organic compounds and nitrogen oxides, under the conditions of ultraviolet light, it will undergo a series of photochemical chain reactions to generate ozone, which will lead to an increase in the surface ozone concentration. This also shows that the governance process has a certain effect, but it also needs to continue to improve the system structure and improve structural stability.

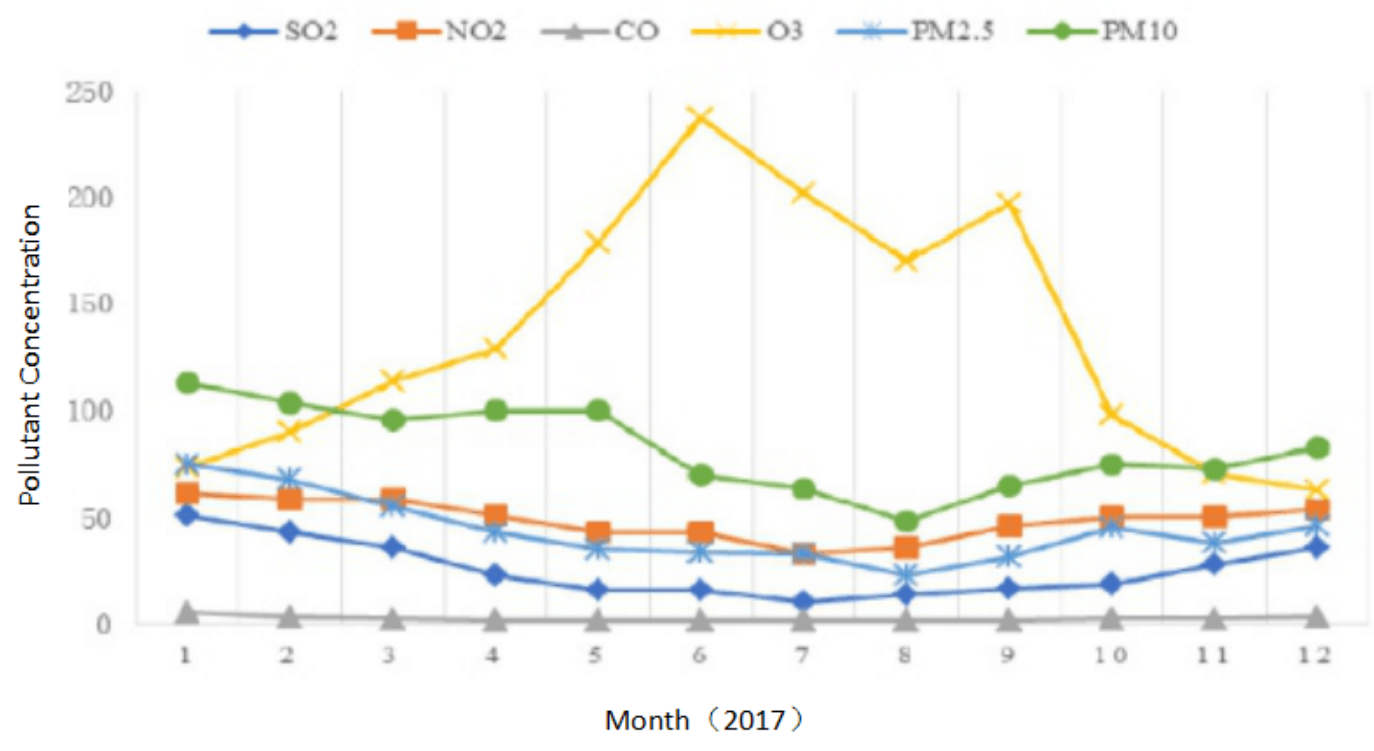

Figure 1: Trends of Air Pollutants in A Certain Area (2017)

\subsection{Reasonable Planning of Ecological Function Zones}

Environmental function zoning is a concept proposed in recent years in order to determine the goals with different environmental functions for environmental planning and environmental management. For areas with high environmental requirements in cities, corresponding protection zones should be established to strengthen maintenance and management.

According to different uses, environmental functional zoning can usually be divided into the following categories: (1) Functional zoning according to the development potential of the environment: for example, economic development zones, free trade zones, green spaces, etc.(2) According to social economic characteristics and future development trends can be divided into residential areas, scientific and technological development areas, industrial development areas, education areas, etc. (3) Divide functional areas according to climate, geographic location and other characteristics.(4) Divide functional areas according to administrative scope, this method It can not only show the geographical characteristics of the environment, but also show the economic characteristics of society. This method is more reasonable. (5) According to the existing environmental conditions, the city is divided into key protection areas, general protection areas, pollution control areas, and key remediation areas.

\subsection{Estimation of Atmospheric Environmental Capacity}

Generally speaking, the natural environment has a certain degree of self-purification ability. This kind of 
self-purification ability within a certain range is called environmental capacity. It can be seen that in urban planning, we need to combine the formulation of environmental quality standards with the local environmental capacity, carry out the evaluation of the impact of industrial planning on the local environment, and promote urban construction and industrial layout on the premise of meeting the environmental self-purification ability.

Specifically, in order to achieve a reasonable layout and optimize the industrial structure, we must first analyze the environmental quality of the planned area. At the same time, we also need to calculate and build an environmental carrying capacity model of the area, and finally assess the atmospheric environmental capacity of the area. Secondly, we must simulate the future development scenarios of the region and evaluate the environmental carrying capacity. Finally, based on the actual local social and economic development trends, the bearing capacity of the atmospheric environment and its relative residual rate are calculated. Through the analysis of the remaining rate of the remaining environmental carrying capacity, we can provide guidance for future planning at a higher level, so as to ensure that future planning can still ensure the protection of the atmospheric environment.

\section{Conclusion}

The rapid social and economic development has brought serious environmental problems and threatened the subsequent development of the city. Therefore, we must add consideration to the problem of air pollution in urban planning. Only in this way can we fundamentally solve and suppress the further deterioration of the air problem. For future urban planning, we need to make sustainable development a guideline. At the same time, we also need to adopt reasonable industrial planning, functional zoning, the introduction of science and technology, and the concept of advocating green living. Only in this way can we contain the deterioration of the atmospheric environment at the source and improve the living environment of the people.

\section{Acknowledgement}

Treatment of dairy wastewater by reverse osmosis membrane and ultrafiltration Project No: HDFHX170105

\section{References:}

1. Wang Xuanya. Legal coordination mechanism for the integration of urban planning and air pollution prevention and control [N]. Consumer Daily, 2015-09-03 (A03).

2. Yong Juan, Hu Wantai, Song Siman, Li Gang. Research on urban planning factors and interactive relationship model affecting air pollution [C]. Chinese Association of Science and Technology. Proceedings of the Second Mountain Town
Sustainable Development Expert Forum. China Science and Technology Association: Chinese Society of Urban Planning, 2013: 205-212.

3. Zhou Yajie, Zhou Huan. Solving urban air pollution problems in urban planning [J]. Henan Science and Technology, 2013 (10): 186.

4. Chen Yunting, $\mathrm{Xu}$ Jiangang, Tong Jianghua, $\mathrm{Wu}$ Yang. Industrial air pollution simulation in urban planning__ Taking Nanjing as an example[C]. Chinese Urban Planning Society. Urban and rural planning from the perspective of ecological civilization-2008 China Proceedings of the annual meeting of urban planning. Chinese Urban Planning Society: Chinese Urban Planning Society, 2008: 4307-4314.

5. Liu Huanbin, Wang Hengming. Shandong Province Air Pollution Coefficient Distribution and Its Application in Urban Planning [J]. Shandong Meteorology, 2005(02): 29-30. 